\title{
Familial mutation in Caffey disease with reduced penetrance: A case report
}

\author{
Özmert M.A. Özdemir ${ }^{1}$, Hazal Tancer-Elçi², Aziz Polat² ${ }^{2}$ İnci Güçtürk², Emre Tepeli \\ Selcan Zeybek ${ }^{3}$, Akif Ayaz ${ }^{3}$ \\ ${ }^{1}$ Division of Neonatology, ${ }^{2}$ Department of Pediatrics, and ${ }^{3}$ Department of Medical Genetics, Pamukkale University Faculty \\ of Medicine, Denizli, Turkey. \\ E-mail: drozmert@gmail.com \\ Received: 5th April 2016, Revised: 15th July 2016, Accepted: 2nd August 2016
}

SUMMARY: Özdemir ÖMA, Tancer-Elçi H, Polat A, Güçtürk İ, Tepeli E, Zeybek $S$, Ayaz A. Familial mutation in Caffey disease with reduced penetrance: A case report. Turk J Pediatr 2016; 58: 650-653.

Caffey disease is a rare condition of early infancy, characterized by soft tissue swelling, bone lesions, and hyperirritability. Its typical radiological finding is periosteal new bone formation. It can be sporadic or inherited in an autosomal dominant manner. There is no specific treatment. In symptomatic cases, non-steroidal anti-inflammatory drugs such as ibuprofen, indomethacin, or naproxen can be used. This is a report of an infant who presented with restlessness, irritability, and swelling over his shins, diagnosed as Caffey disease. Although there was no family history, the genetic analysis revealed heterozygous missense mutation (c.3040C $>$ T) in type-I-collagen-alpha-1-chain gene in the patient in addition to his mother, grandmother, aunt, and cousin. After indomethacin therapy, the complaints of the patient were completely resolved and his bone lesions were significantly improved. This case report is a familial form of Caffey disease from Turkey, with proven heterozygous mutation in the patient and the family members.

Key words: Caffey disease, COL1A1, mutation, infant.

Caffey disease, also known as infantile cortical hyperostosis (ICH) is a rare condition of early infancy which is considered to be a benign and self-limiting inflammatory disease of uncertain etiology. It is characterized by soft tissue swelling, bone lesions, and hyperirritability. It may be misdiagnosed or diagnosis may be delayed because of the wide range of bone diseases that it can mimic. The typical radiological feature is a marked periosteal new bone formation. It can be sporadic or inherited in an autosomal dominant manner ${ }^{1-6}$. This is a report of an infant who presented with restlessness, irritability, and swelling over his shins and was diagnosed with Caffey disease. There was no family history but the genetic analysis revealed heterozygous mutation in type I collagen alpha-1 chain (COL1A1) gene in the presented patient in addition to some members of the patient's family.

\section{Case Report}

A fifty-two-day-old boy was referred to our hospital, with a complaint of restlessness and swelling over his shins noticed by the mother one week before. He was born after a normal pregnancy period, full term by normal vaginal delivery and his birth weight was $2.8 \mathrm{~kg}$. Routine prenatal follow-up and ultrasounds were normal. There was no history of child abuse, trauma and fever. He was only breast fed and was having vitamin-D supplement 400 $\mathrm{U} /$ day. The mother and father was the first cousin once removed. There was a suspicion of bone disease in his cousin in infancy, but the exact diagnosis was not known by the family. It was also learned that the cousin was healthy now with no bone deformations. There was no other family history with a major medical problem. 
On physical examination of the patient, he was irritable, crying when lower limbs were touched. All vital signs were normal. His weight, length and head circumference percentiles were 5075p, 10-25p and 10-25 p, respectively. There was bilateral swelling and tenderness over his shins, without any erythema or ecchymosis. He kept his lower limbs at flexion posture. The remaining systemic examinations were normal.

Laboratory investigations showed elevated levels of erythrocyte sedimentation rate (ESR, 60 $\mathrm{mm} / \mathrm{hr}$ ) and alkaline phosphatase (537 IU/L), and a mild thrombocytosis $\left(572.000 / \mathrm{mm}^{3}\right)$. Peripheral blood smear showed hypo chromic red cells, target cells and thrombocytosis with no other abnormality. Serum C-reactive protein (CRP), calcium, amylase, parathormone, 25-OH vitamin-D levels and the other biochemical and hematological evaluations were normal. Patient's and the mother's serology for syphilis (VDRL) was nonreactive. Serum immunoglobulin and urine analyses were normal, and serology for viral infections was negative. Radiological examination showed cortical thickening, sub periosteal reaction and new bone formation in the mandible, bilateral clavicles and long bones (Fig. 1a and b).

With all of these clinical, physical examination, laboratory, and radiological findings, the patient was diagnosed with Caffey disease. In genetic investigation, heterozygous missense mutation (c.3040C > T) in COL1A1 gene was detected in our patient with sequence analyzes method (Fig. 2). When the family was scanned, the father's and grandfather's analysis revealed normal results, whereas in the mother, grandmother, and aunt who had no history of any bone diseases, and in the cousin who had a bone disease history in infancy, the same mutation was detected and it was shown on pedigree of the patient (Fig. 3). On radiological examination of the patient's family members, there was no abnormal findings.

With the diagnosis of Caffey disease, the patient was commenced ibuprofen treatment with a dose of $10 \mathrm{mg} / \mathrm{kg}$ three times a day. At the fourth week of ibuprofen treatment, there was no clinical improvement, therefore ibuprofen treatment was aborted and indomethacin (3 $\mathrm{mg} / \mathrm{kg} /$ day) was started. At his fourth month of the follow-up period, the patient showed no restlessness or irritability, and the swelling of extremities was resolved. The control X-rays showed significant regression in periosteal reaction and new bone formation in mandible and lower limb bones with minimal angulations, complete resolution in upper limb bones (Fig. $4 \mathrm{a}$ and $\mathrm{b}$ ).

\section{Discussion}

Caffey disease or $\mathrm{ICH}$ is a rare condition that was first described by Caffey and Silverman in $1945^{1}$. It is a self-limiting inflammatory disease that mostly affects infants before 6 months of age and disappears during childhood. It is characterized by a triad of soft tissue swelling, bone lesions, and systemic symptoms such as fever and hyperirritability, as shown in our patient ${ }^{1-4}$. Erythema and increased warmth of the overlying tissues may be seen. Movement of the involved part is limited by pain as in our case, even leading to pseudo paralysis ${ }^{7}$. There are two forms of Caffey disease that have been described, the classical infantile form (ICH), and lethal prenatal form ${ }^{1-3,6}$.

In the classical form, the initial symptom is hyperirritability, followed by soft tissue swelling, tenderness, and painful movement of

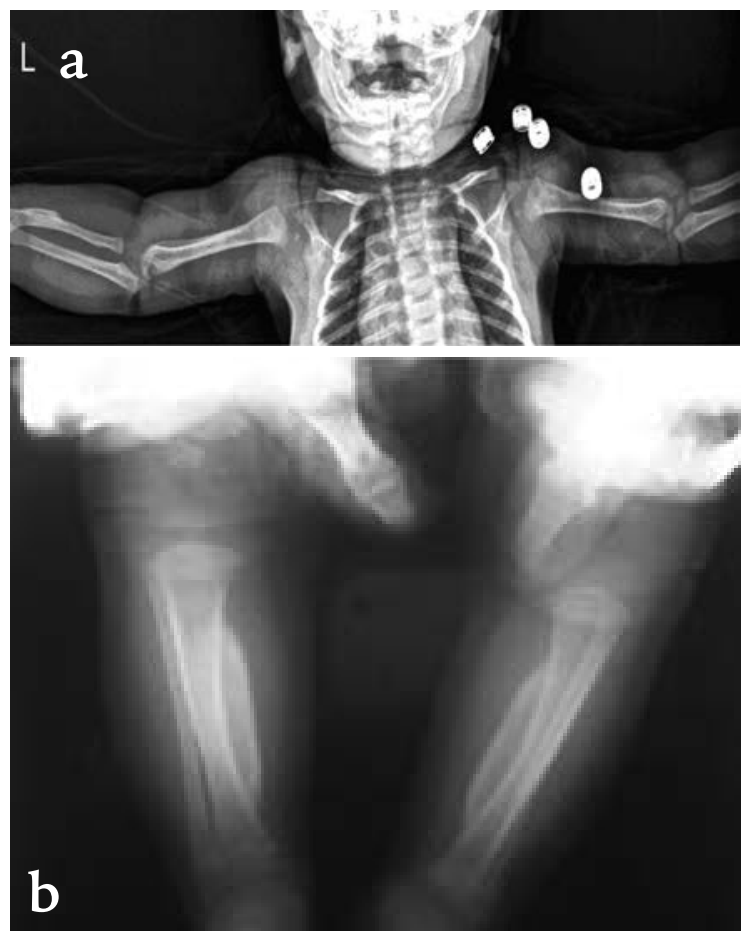

Fig. 1. X-ray shows; (a) hyperostosis of bilateral clavicles, upper limbs and mandible; (b) hyperostosis of bilateral lower limbs. 


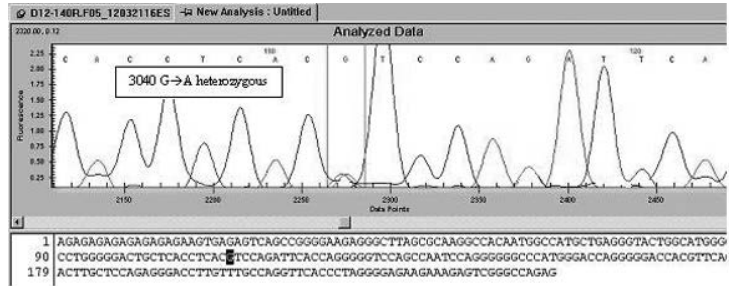

Fig. 2. Sequence analysis of the patient shows heterozygous mutation in COL1A1.

the affected area. Systemic symptoms including fever and anorexia can be seen ${ }^{8}$. Mandible is the most commonly involved site followed by scapula, clavicle, ribs and long bones ${ }^{9}$. Tibia is the most often involved in familial cases $^{6}$. The involvement can be symmetrical or asymmetrical2,8. Some uncommon features like facial nerve palsy, Erb's palsy, dysphagia, nasal obstruction and proptosis have been reported $^{3,10,11}$. In our case, mandible, bilateral clavicles, bilateral upper and lower long bones were involved, and no uncommon findings were present.

Most infants with infantile cortical hyperostosis are diagnosed by physical examination; however, radiological examination is an important diagnostic method to confirm the presence of bone changes and soft tissue swelling ${ }^{2-4}$. The characteristic X-ray findings include cortical new bone formation beneath the areas of soft tissue swelling, causing abnormal thickening of cortical bone (hyperostosis), symmetrical or asymmetrically ${ }^{2-4,8}$. The characteristic bone lesions were also shown in the present patient.

The etiology still remains unknown ${ }^{3}$. Most of the cases are reported to be sporadic, but a few familial cases with autosomal dominant

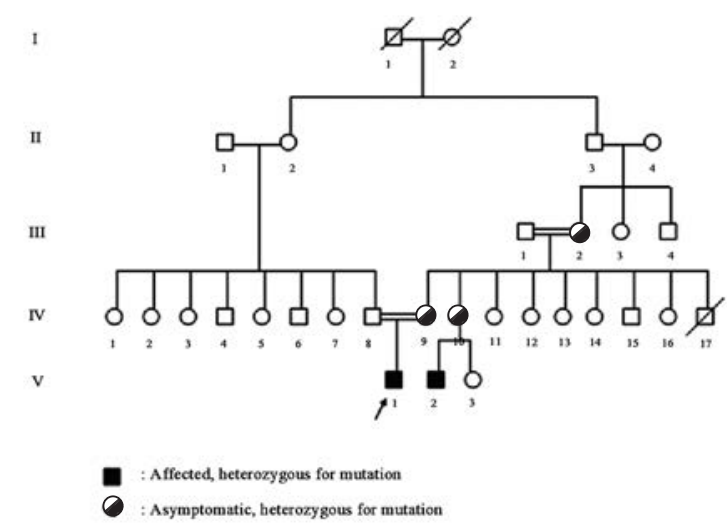

Fig. 3. Pedigree of the patient. inheritance have been reported $5,6,12$. A heterozygous missense mutation, c.3040C $>\mathrm{T}$, in the type I collagen alpha- 1 chain gene (COL1A1, 17q21) has been reported in families with the autosomal dominant form of Caffey disease ${ }^{5}$. In our case, the genetic analyses revealed normal results in the father and grandfather, while in the patients, mother, grandmother, aunt and cousin, heterozygous mutation was detected. The mother, grandmother and aunt had no history of irritability or any swelling tissue during their infant's period, but it is known that some cases can be asymptomatic. This situation can be explained by two facts that, obligate carriers can lack cortical hyperostosis due to reduced penetrance, or the family might overlook the sign and symptoms of the disease. It has been reported that, the affected
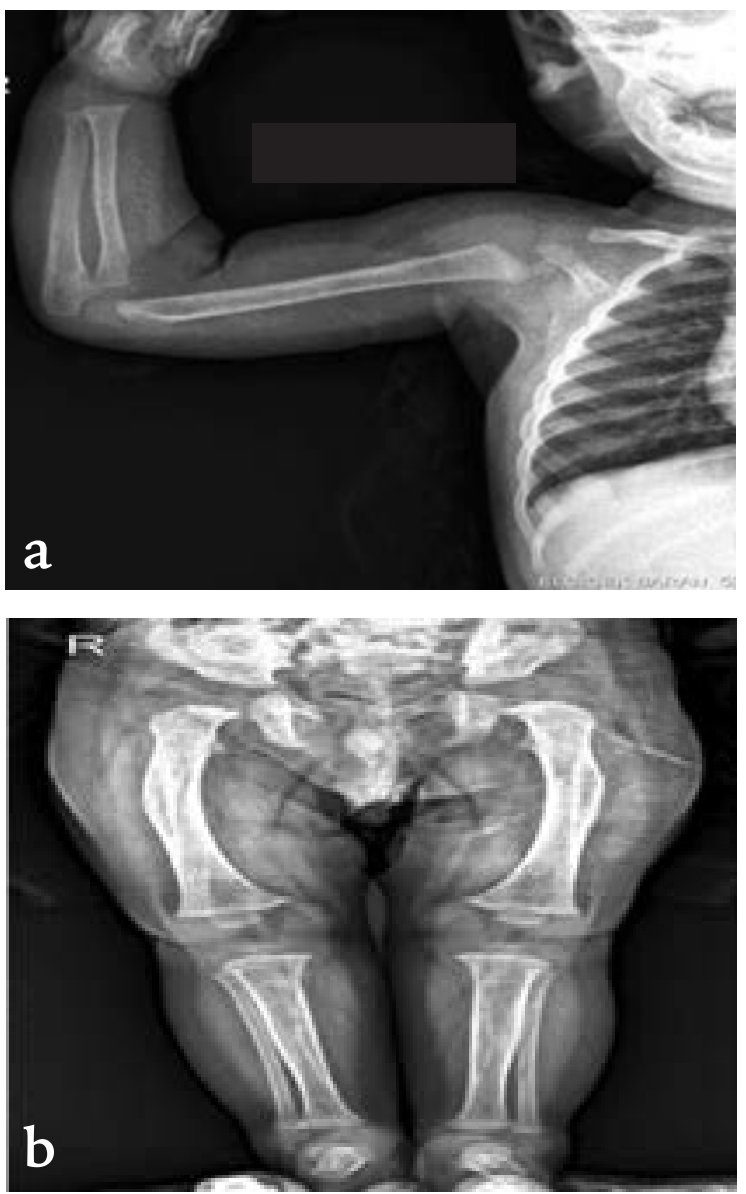

Fig. 4. X-ray shows; (a) complete resolution in periosteal reaction and new bone formation in the upper limb bones, and; (b) significant regression in the lower limb bones with minimal angulation. 
individuals could have angular deformities of the long bones and short stature which may occur during follow-up. Minimal angular deformities in the lower limb bones of our patient were also detected, which improved clinically during follow-up.

Caffey disease can be misdiagnosed or diagnosis may be delayed because of the wide range of diseases that it can mimic, including child abuse, trauma, syphilis, scurvy, hyper vitaminosis A, prostoglandin-E1 administration, Ewing sarcoma, and metastatic bone tumors $3,6,13$. In our case, trauma and child abuse were excluded because the patient's history and physical examination was normal. Bone tumors were not considered according to radiological and biochemical findings. Serology for syphilis was nonreactive. The patient was only breast fed and had vitamin D supplement only, so that scurvy and hypervitaminosis A was excluded. In addition, the parathormone and $25-\mathrm{OH}$ vitamin-D levels of the patient were normal. There was no history of congenital heart disease requiring prostoglandin-E1 administration. In this disease, laboratory findings include elevated ESR, and in some patients, high alkaline phosphatase, thrombocytosis, anemia and raised immunoglobulin levels ${ }^{3,9}$. The presented patient had elevated ESR, thrombocytosis and high alkaline phosphatase, but the levels of immunoglobulin and hemoglobin were normal.

Caffey disease is a self-limiting disorder that resolves on its own without treatment, usually within six to nine months $s^{3,13}$. Management of Caffey disease is essentially palliative, however, in symptomatic and painful cases non-steroidal anti-inflammatory drugs such as ibuprofen, indomethacin, or naproxen can be used ${ }^{3,9,14}$. In our case, ibuprofen was used firstly but there was no improvement in the patient's symptoms. After 4 weeks of ibuprofen usage, indomethacin was started and the outcome was satisfactory.

As a result, from Turkey, Caksen et al. ${ }^{4}$ have reported a sporadic case with Caffey disease. However, the presented patient is a case report of familial form of Caffey disease from Turkey, with proven heterozygous mutation in the patient and the family members. This disease should be considered in the differential diagnosis of the presence of painful limb swelling and cortical hyperostosis in a young infant.

\section{REFERENCES}

1. Caffey J, Silverman W. Infantile cortical hyperostosis, preliminary report of a new syndrome. Am J Roentgenol Rad Therapy 1945; 54: 1-16.

2. Caffey J. Infantile cortical hyperostosis; a review of the clinical and radiographic features. Proc R Soc Med 1957; 50: 347-354.

3. Kutty N, Thomas D, George L, John TB. Caffey disease or infantile cortical hyperostosis: A case report. Oman Med J 2010; 25: 134-136.

4. Caksen H, Cesur Y, Odabas D, Aslan H, Rastgeldi L. A case of infantile cortical hyperostosis. J Nihon Med Sch 2001; 68: 442-443.

5. Cho TJ, Moon HJ, Cho DY, et al. The c.3040C > $\mathrm{T}$ mutation in COL1A1 is recurrent in Korean patients with infantile cortical hyperostosis (Caffey disease). J Hum Genet 2008; 53: 947-949.

6. Gensure RC, Mäkitie O, Barclay C, et al. A novel COL1A1 mutation in infantile cortical hyperostosis (Caffey disease) expands the spectrum of collagenrelated disorders. J Clin Invest 2005; 115: 1250-1257.

7. Wagner JM, Solomon A. Hyperostosis corticalis infantalis (Caffey's disease). S Afr Med J 1969; 43: 754-755.

8. Glorieux FH. Caffey disease: an unlikely collagenopathy. J Clin Invest 2005; 115: 1142-1144.

9. Mishra P, Panda SS, Tripathy M, Panda M, Das RR. Infantile cortical hyperostosis: two cases with varied presentations. J Clin Diagn Res 2014; 8: PJ01-2.

10. Challapalli M, Cunningham DG, Varnado SC. Infantile cortical hyperostosis and facial nerve palsy. Int J Pediatr Otorhinolaryngol 1998; 43: 175-178.

11. Sheppard JJ, Pressman H. Dysphagia in Infantile cortical hyperostosis (Caffey's disease): A case study. Dev Med Child Neurol 1988; 30: 111-114.

12. Bernstein RM, Zaleska DJ. Familial aspects of Caffey's disease. Am J Orthop (Belle Mead NJ) 1995; 24: 777 781.

13. Ranganath P, Laine CM, Gupta D, Makitie O, Phadke SR. COL1A1 mutation in an Indian child with Caffey disease. Indian J Pediatr 2011; 78: 877-879.

14. Lo HP, Lau HY, Li CH, So KT. Infantile cortical hyperostosis (Caffey disease): a possible misdiagnosis as physical abuse. Hong Kong Med J 2010; 16: $397-$ 399. 